\title{
Ancestral polymorphism at the major histocompatibility complex (MHCII $)$ in the Nesospiza bunting species complex and its sister species (Rowettia goughensis)
}

Alexandra Jansen van Rensburg ${ }^{1 *}$, Paulette Bloomer ${ }^{1 \dagger}$, Peter G Ryan ${ }^{2 \dagger}$ and Bengt Hansson ${ }^{3 *}$

\begin{abstract}
Background: The major histocompatibility complex (MHC) is an important component of the vertebrate immune system and is frequently used to characterise adaptive variation in wild populations due to its co-evolution with pathogens. Passerine birds have an exceptionally diverse MHC with multiple gene copies and large numbers of alleles compared to other avian taxa. The Nesospiza bunting species complex (two species on Nightingale Island; one species with three sub-species on Inaccessible Island) represents a rapid adaptive radiation at a small, isolated archipelago, and is thus an excellent model for the study of adaptation and speciation. In this first study of MHC in Nesospiza buntings, we aim to characterize $M H C \| \beta$ variation, determine the strength of selection acting at this gene region and assess the level of shared polymorphism between the Nesospiza species complex and its putative sister taxon, Rowettia goughensis, from Gough Island.
\end{abstract}

Results: In total, 23 unique alleles were found in 14 Nesospiza and $2 R$. goughensis individuals encoding at least four presumably functional loci and two pseudogenes. There was no evidence of ongoing selection on the peptide binding region (PBR). Of the 23 alleles, 15 were found on both the islands inhabited by Nesospiza species, and seven in both Nesospiza and Rowettia; indications of shared, ancestral polymorphism. A gene tree of Nesospiza MHCII $\beta$ alleles with several other passerine birds shows three highly supported Nesospiza-specific groups. All $R$. goughensis alleles were shared with Nesospiza, and these alleles were found in all three Nesospiza sequence groups in the gene tree, suggesting that most of the observed variation predates their phylogenetic split.

Conclusions: Lack of evidence of selection on the PBR, together with shared polymorphism across the gene tree, suggests that population variation of $\mathrm{MHCl} \beta$ among Nesospiza and Rowettia is due to ancestral polymorphism rather than local selective forces. Weak or no selection pressure could be attributed to low parasite load at these isolated Atlantic islands. The deep divergence between the highly supported Nesospiza-specific sequence Groups 2 and 3, and the clustering of Group 3 close to the distantly related passerines, provide strong support for preserved ancestral polymorphism, and present evidence of one of the rare cases of extensive ancestral polymorphism in birds.

\section{Background}

Understanding the principals that govern the generation and maintenance of functional genetic diversity is fundamental to evolutionary biology. Large reductions in

\footnotetext{
* Correspondence: alexjvr@gmail.com; bengt.hansson@biol.lu.se ${ }^{\dagger}$ Equal contributors

'Molecular Ecology and Evolution Program, Department of Genetics, University of Pretoria, Private bag X20, Hatfield 0028, South Africa ${ }^{3}$ Molecular Ecology and Evolution Lab, Department of Biology, Lund University, SE-22362 Lund, Sweden

Full list of author information is available at the end of the article
}

population size, through bottleneck or founder events, result in a loss of genetic diversity [1] which may affect the ability of populations to adapt and survive in changing environments [1,2]. However, genes of ecological adaptive importance may maintain variation through a severe reduction in population size through processes such as balancing selection [3,4]. The Major Histocompatibility Complex (MHC) is such a functional locus, and has been extensively studied in both model and non-model species [5-7].

\section{Biomed Central}


The MHC is a multigene family involved in the vertebrate immune response [8], and is the most polymorphic set of genes known in vertebrates $[9,10]$. MHC variation is driven by an arms race between host and pathogen, where balancing selection maintains alleles in the population. An extensive repertoire of alleles enables the population to respond rapidly to changing or novel pathogens [11-13]. The highly variable peptide binding region (PBR) encoded by $\mathrm{MHC}$ class II $\beta$ exon 2 $(\mathrm{MHCII} \beta)$ ensures the binding of a large number of conformationally different peptides [8]. The PBR of MHC molecules is involved in antigen recognition and as such may be under strong balancing selection when compared with the non-PBR sites [14]. Although the major driving force behind $\mathrm{MHC}$ diversity is hostpathogen co-evolution $[11,15]$, sexual selection and selection against deleterious mutations also play a role in the maintenance of $\mathrm{MHC}$ variation [16-18].

Like many multi-gene families, MHC is governed by the birth-and-death model of evolution where new genes are generated through gene duplication. Some of these genes are maintained for long periods and even through population divergence events, while others lose function (pseudogenes) or are lost completely. MHC variation is also governed by gene conversion, where homologous recombination occurs between duplicated genes (paralogous genes), thus homogenising sequences between different loci $[6,19]$. In passerine birds, the $\mathrm{MHC}$ is characterised by multiple gene copies, pseudogenes and long introns, and is exceptionally diverse and complex compared to other birds and vertebrate species [20-22]. Gene duplication events of MHC can be traced phylogenetically in most lineages, because duplicated genes evolve independently. This can be seen in the phylogenetic grouping of orthologous genes, rather than in a species-specific grouping [19,23,24]. Alternatively, recent duplication and concerted evolution of genes (through gene conversion) can result in species-specific clustering $[6,22,25,26]$. Due to the high rate of gene duplication and loss, and the confounding effect of gene conversion, it is notoriously difficult to re-construct avian $\mathrm{MHC}$ phylogenies [6].

Following a bottleneck or founder event, the genetic diversity of a population is reduced to only a subset of the original variation. As the population adapts to its new environment, the MHC allelic diversity will be made up of a combination of ancestral polymorphism and novel genetic variation. Trans-species evolution [27] or ancestral polymorphism [28] refers to the long-term maintenance of ancestral alleles in populations and species $[29,30]$. This process is governed by balancing selection [31] and is seen when related species or subspecies share similar or the same MHC alleles despite local selection pressure. This pattern is common in mammals which do not often show concerted evolution, thus orthologous loci can be recognized between distantly related taxa such as mice and humans [24]. The high levels of concerted evolution in birds often make it difficult to distinguish between orthologous and paralogous loci [25], although isolated cases have been reported e.g. [5,32]. Novel genetic diversity is introduced in populations either through dispersal or mutations. Mutational processes include gene duplication, point mutations and gene conversion e.g. [26,33]. Gene conversion is known to occur frequently in birds at the highly duplicated MHC genes $[6,26,34,35]$. The rate of gene conversion has been shown to be far greater than that of point mutations, thus may be a very important mechanism for generation of variation in bottlenecked populations $[9,26]$.

In the present study, we assess MHC variation in the Nesospiza bunting species complex and its putative sister taxon, Rowettia goughensis. Evaluation of the MHC in Nesospiza and $R$. goughensis is interesting for several reasons. Nesospiza and R. goughensis are considered sister taxa and are presumed to have arrived at Tristan da Cunha and nearby Gough Island with the same colonization event [36]. Mitochondrial cytochrome $b$ sequences are reciprocally monophyletic between island systems, and neutral microsatellite markers show substantial genetic differentiation between species [37,38]. It is thus interesting to compare the MHC differentiation and allele sharing in Nesospiza and R. goughensis and determine the level of ancestral polymorphism between these species. Further, Nesospiza buntings have undergone an ecological adaptive radiation in parallel on two islands [37]. Both Nightingale and Inaccessible islands are inhabited by large- and small-billed Nesospiza buntings. The two species on Nightingale Island ( $N$. questi and $N$. wilkinsi) co-occur with little, if any, interbreeding, probably due to the availability of two discrete seed sizes within a single habitat. Inaccessible Island has three lineages of $N$. acunhae buntings: large-billed $N$. a. dun$n e i$, and two colour morphs of the small-billed bunting, $N$. a. fraseri and $N$. a. acunhae [37,39]. Hybridisation occurs between all three forms across an ecotone on the eastern plateau of Inaccessible Island. This is probably due to a large variation of seed sizes occurring at low densities, which favours greater diversity in bill-sizes [37]. A single Nesospiza species inhabited the main island of Tristan, but was driven to extinction shortly after the arrival of humans at the archipelago. Genetic structure analysis based on neutral microsatellite markers show little or no hybridization between species on Nightingale, and strong differentiation between Nightingale Nesospiza and those on Inaccessible Island [37,38]. Despite ongoing hybridization on Inaccessible Island, a strong association has been found between bill morphology, habitat choice and genetic differentiation suggesting that both natural 
and sexual selection may maintain differentiation $[37,38]$. Thus, it is possible that these selective pressures will result in species-specific patterns of MHC variation. However, an alternative hypothesis is that balancing selection has maintained most of the MHC variation across the species complex. Here we aim to 1) test for signatures of selection at the MHCII $\beta$ in Nesospiza buntings, and 2) investigate the extent of ancestral polymorphism between Nesospiza, its putative sister taxon Rowettia goughensis, and other passerine species $[5,32,34,35,40,41]$.

\section{Results}

\section{PCR amplification success and nucleotide diversity}

In total, 508 sequences of expected length (159 bp) were obtained from 14 Nesospiza from the Tristan da Cunha archipelago (10 from Inaccessible and 4 from Nightingale) and two Rowettia goughensis from Gough Island (see Figure 1). Only sequences that were found in two or more individuals were included (396 sequences), and among these, 23 unique alleles were identified (Figure 2; Additional file 1 Table S1). Since the MHC complex contains several paralogous loci, alleles cannot be assigned to a particular locus. This prevents the use of the standard nomenclature of $\mathrm{MHC}$ alleles [42], and therefore alleles were named NesoO1 - Neso23. No stop codons or frameshift mutations were present in any of these alleles, although one of the sequences (Neso02) contained an in-frame two codon insert, resulting in a 165 bp sequence. BLAST analysis indicated high similarity (87-96\%, with coverage of $80-98 \%$ ) of 21 alleles (Neso01-Neso21) to functional passerine MHCII alleles, whereas Neso22 and Neso23 had higher similarity (92$93 \%$, with $98 \%$ coverage) to passerine pseudogenes.

Each individual Nesospiza contained 3-7 unique presumably functional (i.e. excluding known pseudogenes Neso22 and Neso23) alleles of MHCII (average \pm SD: $4.63 \pm 0.99$ ). Assuming all loci to be heterozygous, the minimum number of MHCII $\beta$ loci that must be present in Nesospiza is four. This is similar to what has been observed in most passerine species (3-7 loci), with the exception of common yellowthroat (Geothlypis trichas) (20 loci), which has particularly high levels of gene duplication [43]. A regression analysis performed to determine if the number of alleles sampled approached the maximum for each individual showed that the number of

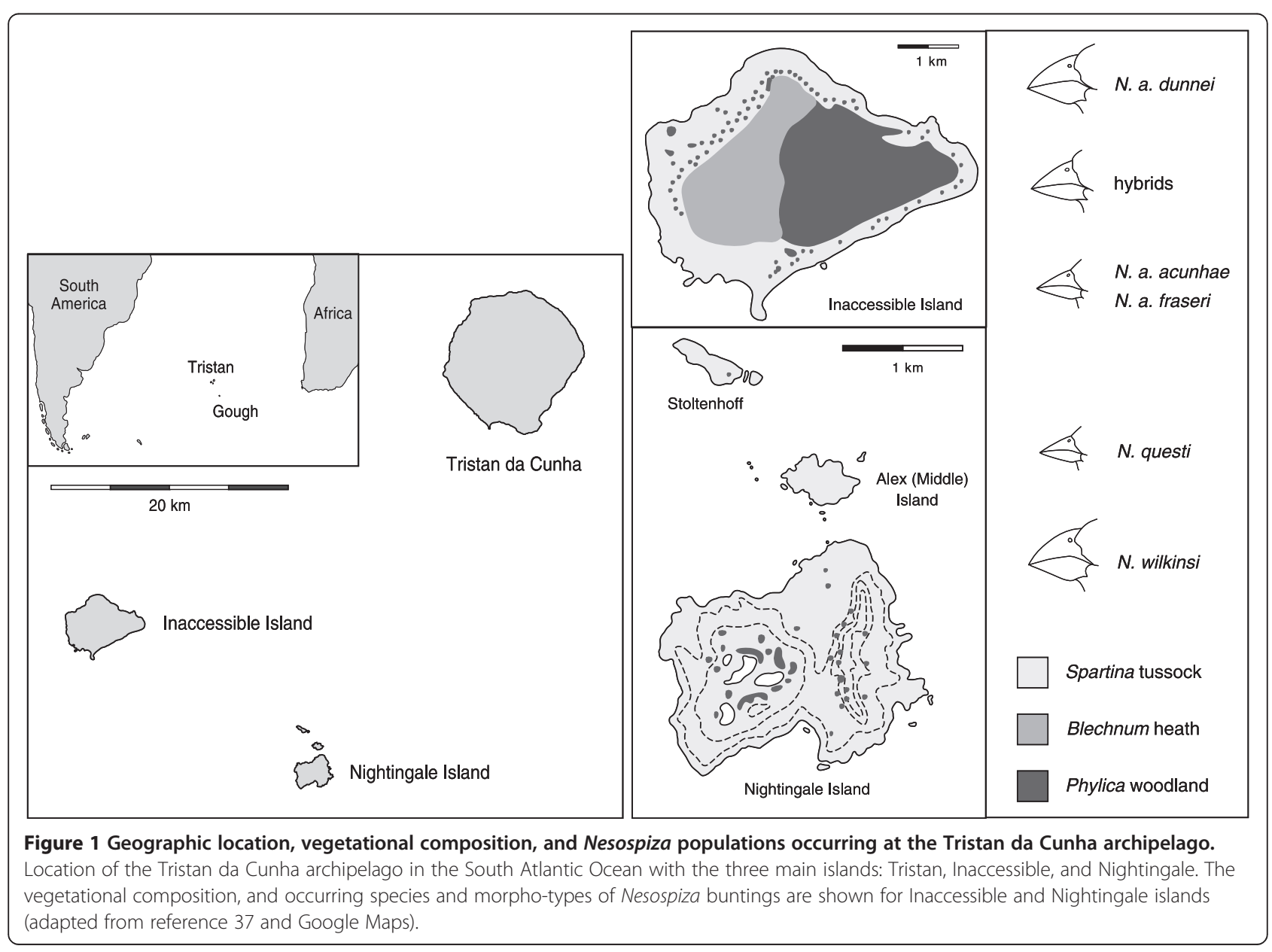




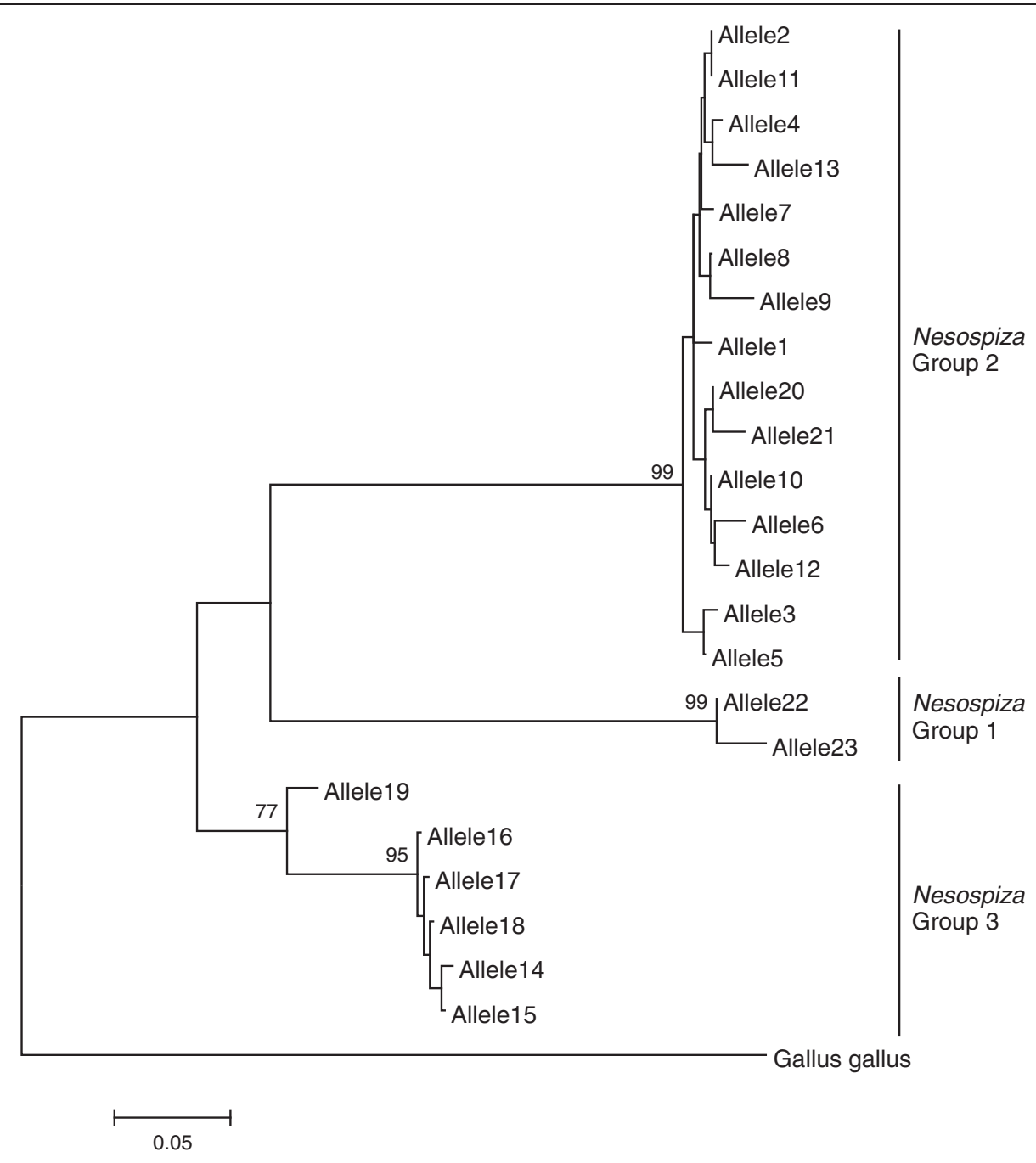

Figure 2 Nesospiza MHCII $\beta$ gene tree. Neighbour-Joining tree showing the three well supported Nesospiza MHCII exon 2 allele clusters. Of the 23 alleles, 21 were found in the N. acunhae individuals on Inaccessible Island (Neso 1-8, 10-13, 15-23), 14 in the N. wilkinsi and N. questi on Nightingale (Neso 1, 3, 4, 7, 9, 11, 13-15, 17, 18, 20, 22, 23), and 7 in R. goughensis (Nes05, 9, 13-15, 17, 23). Bootstrap support <70\% are not shown.

alleles did not plateau for 13 of the 16 individuals as the number of sequence clones increased (data not shown); thus, it is likely that more than four MHCII $\beta$ loci are present in Nesospiza.

Of the 23 alleles, 21 were found in the $N$. acunhae individuals on Inaccessible Island (Neso1-8, 10-13, 1523), 14 in the $N$. wilkinsi and $N$. questi on Nightingale (Neso1, 3, 4, 7, 9, 11, 13-15, 17, 18, 20, 22, 23), and 7 in R. goughensis (Neso5, 9, 13-15, 17, 23). The nucleotide diversity $(\pi)$ of putatively functional alleles (i.e. excluding the pseodogenes, Neso22 and Neso23) was 0.11 in $N$. acunhae on Inaccessible Island (data from 19 alleles in 10 individuals), 0.11 in $N$. wilkinsi on Nightingale (data from 8 alleles in 2 individuals), and 0.07 in $N$. question Nightingale (data from 7 alleles in 2 individuals). The nucleotide diversity (0.04) found in $R$. goughensis (data from the 6 alleles in 2 individuals).

\section{Selection and recombination}

The PBR was identified after alignment with the human HLA-DRB*04 amino acid sequence. Traditional selection statistics did not uncover any statistically significant selection patterns (Tajima's D $=0.61, \mathrm{p}>0.10 ; \mathrm{Fu} \&$ Li's $D^{*}=0.30, \mathrm{p}>0.10 ; \mathrm{Fu} \&$ Li's $\left.\mathrm{F}^{*}=0.46, \mathrm{p}>0.10\right)$. The sampled populations showed no evidence of selection at the either the PBR or non-PBR regions (Table 1). Null models were supported by likelihood ratio tests, with only one site likely to be under positive selection (Table 2). Tests for recombination in RDP3 Beta 27 revealed no significant recombination events. 
Table 1 Proportion of non-synonymous $\left(d_{N}\right)$ and synonymous $\left(d_{s}\right)$ substitutions in MHCII sequences of Nesospiza and other passerines

\begin{tabular}{|c|c|c|c|c|c|c|c|c|c|}
\hline \multirow[b]{2}{*}{ Comparisons } & \multirow[b]{2}{*}{$\mathrm{N}$} & \multicolumn{4}{|l|}{ PBR } & \multicolumn{4}{|l|}{ Non-PBR } \\
\hline & & $\mathrm{d}_{\mathrm{N}}( \pm \mathrm{SE})$ & $d_{S}( \pm S E)$ & $\mathrm{d}_{\mathrm{N}} / \mathrm{d}_{\mathrm{s}}$ & z-test & $\mathrm{d}_{\mathrm{N}}( \pm \mathrm{SE})$ & $d_{S}( \pm S E)$ & $\mathrm{d}_{\mathrm{N}} / \mathrm{d}_{\mathrm{s}}$ & z-test \\
\hline \multicolumn{10}{|l|}{ Brown et al. [44] } \\
\hline Neso01-23 & 23 & $0.377( \pm 0.146)$ & $0.236( \pm 0.161)$ & 1.60 & n.s. (0.54) & $0.133( \pm 0.029)$ & $0.114( \pm 0.051)$ & 1.17 & n.s. $(0.74)$ \\
\hline Neso01-21 \# & 21 & $0.372( \pm 0.142)$ & $0.234( \pm 0.174)$ & 1.59 & n.s. $(0.59)$ & $0.090( \pm 0.022)$ & $0.097( \pm 0.047)$ & 0.93 & n.s. $(0.90)$ \\
\hline Group1 & 2 & $0.031( \pm 0.031)$ & $0.000( \pm 0.000)$ & $\mathrm{n} / \mathrm{a}$ & n.s. $(0.33)$ & $0.011( \pm 0.011)$ & $0.039( \pm 0.040)$ & 0.28 & n.s. $(0.51)$ \\
\hline Group2 & 15 & $0.009( \pm 0.006)$ & $0.000( \pm 0.000)$ & $\mathrm{n} / \mathrm{a}$ & n.s. $(0.16)$ & $0.028( \pm 0.007)$ & $0.042( \pm 0.030)$ & 0.67 & n.s. (0.68) \\
\hline Group3 & 6 & $0.073( \pm 0.033)$ & $0.036( \pm 0.049)$ & 2.03 & n.s. $(0.51)$ & $0.023( \pm 0.010)$ & $0.014( \pm 0.014)$ & 1.64 & n.s. $(0.62)$ \\
\hline Inaccessible \# & 19 & $0.361( \pm 0.133)$ & $0.222( \pm 0.149)$ & 1.63 & n.s. $(0.53)$ & $0.135( \pm 0.026)$ & $0.115( \pm 0.050)$ & 1.17 & n.s. $(0.74)$ \\
\hline Nightingale $\neq$ & 12 & $0.420( \pm 0.151)$ & $0.281( \pm 0.192)$ & 1.49 & n.s. $(0.60)$ & $0.166( \pm 0.034)$ & $0.118( \pm 0.055)$ & 1.41 & n.s. $(0.43)$ \\
\hline Tristan da Cunha $\neq$ & 21 & $0.377( \pm 0.146)$ & $0.236( \pm 0.161)$ & 1.60 & n.s. (0.54) & $0.133( \pm 0.029)$ & $0.114( \pm 0.051)$ & 1.17 & n.s. $(0.74)$ \\
\hline Rowettia goughensis & 6 & $0.486( \pm 0.188)$ & $0.351( \pm 0.246)$ & 2.59 & n.s. $(0.70)$ & $0.194( \pm 0.040)$ & $0.135( \pm 0.063)$ & 1.44 & n.s. $(0.40)$ \\
\hline \multicolumn{10}{|l|}{ Tong et al. [45] } \\
\hline Neso01-23 & 23 & $0.230( \pm 0.090)$ & $0.125( \pm 0.159)$ & 1.84 & n.s. $(0.61)$ & $0.174( \pm 0.033)$ & $0.143( \pm 0.051)$ & 1.22 & n.s. $(0.61)$ \\
\hline Neso01-21 \# & 21 & $0.100( \pm 0.054)$ & $0.090( \pm 0.147)$ & 0.11 & n.s. $(0.96)$ & $0.151( \pm 0.032)$ & $0.135( \pm 0.055)$ & 1.12 & n.s. $(0.79)$ \\
\hline Group1 & 2 & $0.053( \pm 0.052)$ & $0.000( \pm 0.000)$ & $\mathrm{n} / \mathrm{a}$ & n.s. $(0.33)$ & $0.053( \pm 0.053)$ & $0.000( \pm 0.000)$ & $\mathrm{n} / \mathrm{a}$ & n.s. $(0.32)$ \\
\hline Group2 & 15 & $0.018( \pm 0.019)$ & $0.000( \pm 0.000)$ & $n / a$ & n.s. $(0.38)$ & $0.024( \pm 0.006)$ & $0.033( \pm 0.025)$ & 0.73 & n.s. (0.73) \\
\hline Group3 & 6 & $0.033( \pm 0.022)$ & $0.000( \pm 0.000)$ & $\mathrm{n} / \mathrm{a}$ & n.s. (0.14) & $0.036( \pm 0.013)$ & $0.024( \pm 0.017)$ & 1.50 & n.s. (0.58) \\
\hline Inaccessible $\neq$ & 19 & $0.240( \pm 0.091)$ & $0.126( \pm 0.165)$ & 1.90 & n.s. $(0.57)$ & $0.171( \pm 0.032)$ & $0.139( \pm 0.049)$ & 1.13 & n.s. $(0.59)$ \\
\hline Nightingale $\neq$ & 12 & $0.306( \pm 0.124)$ & $0.152( \pm 0.189)$ & 2.01 & n.s. $(0.54)$ & $0.204( \pm 0.038)$ & $0.152( \pm 0.058)$ & 1.34 & n.s. $(0.44)$ \\
\hline Tristan da Cunha $\neq$ & 21 & $0.230( \pm 0.090)$ & $0.125( \pm 0.159)$ & 1.84 & n.s. $(0.61)$ & $0.174( \pm 0.033)$ & $0.143( \pm 0.051)$ & 1.22 & n.s. $(0.61)$ \\
\hline Rowettia goughensis & 6 & $0.334( \pm 0.123)$ & $0.163( \pm 0.189)$ & 2.05 & n.s. $(0.52)$ & $0.238( \pm 0.045)$ & $0.186( \pm 0.069)$ & 1.28 & n.s. $(0.49)$ \\
\hline New Zealand robin & 41 & $0.339( \pm 0.078)$ & $0.094( \pm 0.059)$ & 3.6 & $<0.005$ & $0.076( \pm 0.019)$ & $0.039( \pm 0.013)$ & 1.95 & n.s. \\
\hline Chatham Island robin & 4 & $0.373( \pm 0.086)$ & $0.135( \pm 0.078)$ & 2.76 & $<0.05$ & $0.099( \pm 0.024)$ & $0.020( \pm 0.014)$ & 5.05 & $<0.005$ \\
\hline Hawaiian honeycreepers & 51 & $0.341( \pm 0.103)$ & $0.076( \pm 0.095)$ & 4.49 & $<0.001$ & $0.121( \pm 0.038)$ & $0.092( \pm 0.053)$ & 1.32 & n.s. \\
\hline Common yellowthroat & 39 & $0.608( \pm 0.120)$ & $0.211( \pm 0.111)$ & 2.88 & $<0.05$ & $0.135( \pm 0.034)$ & $0.137( \pm 0.034)$ & 0.99 & n.s. \\
\hline House sparrow* ${ }^{*}$ & 12 & $0.470( \pm 0.109)$ & $0.123( \pm 0.095)$ & 3.82 & $<0.0001$ & $0.203( \pm 0.047)$ & $0.200( \pm 0.051)$ & 1.02 & n.s. \\
\hline
\end{tabular}

References: New Zealand and Chatham Island robins [34,35], Hawaiian honeycreepers [46], common yellowthroat [43];* Values were calculated from GenBank sequences, ${ }^{\ddagger}$ Does not include the putative pseudogenes Neso22 and Neso23, N= Number of MHC sequences; Brown et al. [44]: PBR=14 amino acids, non-PBR $=41$ amino acids; Tong et al. [45]: $P B R=9$ amino acids, $n o n=P B R=46$ amino acids.

\section{Phylogenetic analysis}

A consensus Neighbour-Joining tree of the 23 Nesospiza alleles showed three highly supported groups, called Nesospiza Group 1 - 3 (Figure 2). The same three Nesospiza groups were highly supported within genealogies for passerine MHCII $\beta$ reconstructed from exon 2 sequences using Bayesian inference (Figure 3). Group 1, containing the Neso22 and Neso23, and a red-winged blackbird pseudogene (Agelaius phoeniceus; APAF030990), form a highly supported, diverged cluster. A second red-winged blackbird pseudogene (APAF030994) and a vegetarian finch (Platyspiza crassirostris) pseudogene (PCAY064469), however, group with other presumably functional passerine $\mathrm{MHC}$ sequences.

Table 2 Parameter estimates and results from four selection models as implemented in CODEML

\begin{tabular}{llll}
\hline Model & Log-likelihood & Parameter estimates & Positively selected sites \\
\hline M1a (nearly neutral) & -576.502 & $p_{0}=0.385, p 1=0.615, \omega 0=0.038$, & Not allowed \\
\hline M2a (positive selection) & $\omega 1=1.000$ & None & \\
\hline & -574.685 & $p_{0}=0.311, p 1=0.628, p 2=0.061$, & Not allowed \\
\hline M7 (beta) & $\omega 0=0.000, \omega 1=1.000, \omega 2=4.847$ & $37 \mathrm{~N}$ \\
\hline M8 (beta and omega) & -576.474 & $p=0.033, q=0.018$ & \\
\hline
\end{tabular}




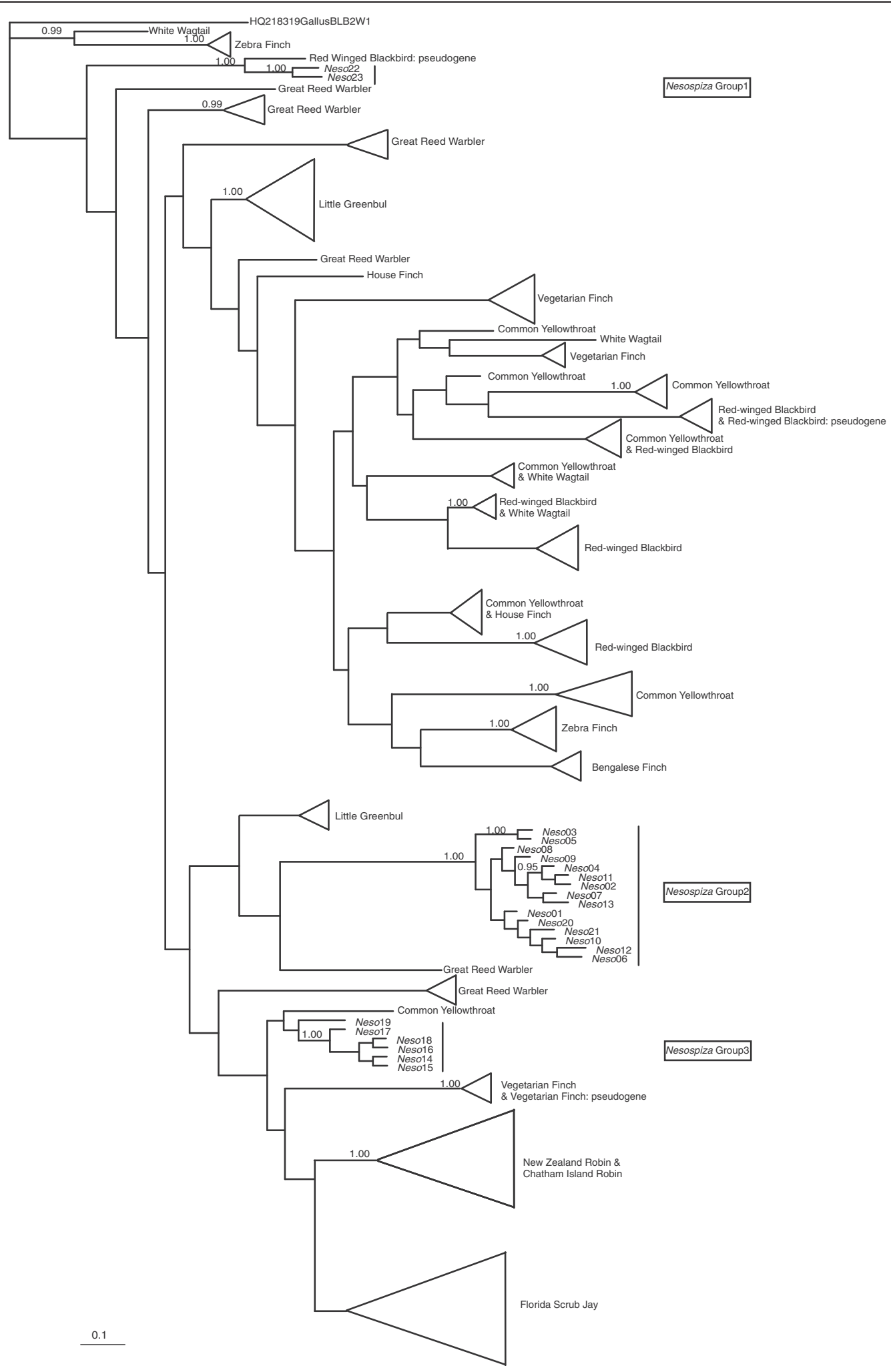

Figure 3 Passerine MHCII gene tree. Gene tree of the MHCIß exon 2 sequences of Nesospiza and sequences of several other passerine species obtained from GenBank. A Bayesian analysis of $159 \mathrm{bp}$ of sequences, with independent mutational models applied to each codon position (Position 1: TIM3ef + I + G; Position 2: TVM + G; Position 3: TPM2uf + G). Bayesian posterior probabilities are indicated at the nodes; values $<0.95$ are not shown. 
Group 2 (Neso01-13, 20-21) is distinct and appears to be a well-supported cluster of presumably functional MHC alleles unique to Nesospiza and $R$. goughensis. Group 3 (Neso14-19), which also contains sequences shared by Nesospiza and R. goughensis, is well supported, but clusters more closely with sequences from the distantly related common yellowthroat, New Zealand robin (Petroica australis), Chatham Island robin (Petroica traverse), Florida scrub jay (Aphelocoma coerulescens) and vegetarian finch. Of the other passerine species, zebra finch, Florida scrub jay, and little greenbul (Andropadus virens; with the exception of one sample) cluster by species or, in the case of New Zealand and Chatham Island robins (Petroica australis), with sister species. Sequences of the great reed warbler (Acrocephalus arundinaceus) are scattered throughout the phylogeny as small groups or single alleles, apart from one supported group divergent from most other passerine sequences. The sequences of several passerines, namely house finch (Carpodacus mexicanus), vegetarian finch, red-winged blackbird, and common yellowthroat, cluster with those of other species throughout the phylogeny.

\section{Discussion}

This study describes $23 \mathrm{MHCII} \beta$ alleles representing at least four functional loci and two pseudogenes in the Nesospiza bunting species complex. Many MHCII $\beta$ alleles were shared between Nesospiza taxa as well as between Nesospiza and its putative sister taxon $R$. goughensis. This pattern of ancestral polymorphism suggests that the observed gene duplications occurred prior to the phylogenetic split of the species, and subsequent unusually low selective pressure at the loci has prevented allelic divergence between species. The MHC nuclear genetic diversity in Nesospiza on Inaccessible $(\pi=0.11)$ was comparable to that of outbred passerine species (e.g. 0.15 in Luscinia svecica; [5]), and despite the low sample size for Nightingale, allele numbers and nucleotide diversity were higher than in the severely bottlenecked Chatham Island robin population (0.05) [35]. We have screened 14 Nesospiza individuals for $\mathrm{MHC}$ variation, which is similar to some previous Passerine $\mathrm{MHC}$ studies using cloning and sequencing e.g. $[34,35,43,47]$. However, because larger sample sizes would have been necessary to cover the variation of each population sufficiently, we will not discuss population-level MHC variation further.

Patterns of both ancestral polymorphism and concerted evolution among Nesospiza and Rowettia populations are evident from our results. Ancestral polymorphism, found here for Nesospiza and R. goughensis, as well as in other species (e.g. great reed warbler, house finch, vegetarian finch, red-winged blackbird and common yellowthroat), can be seen in the sharing of the same or similar alleles between species (Figures 2 and 3). Of the 23 Nesospiza alleles, 15 were found in species from both islands. All seven alleles occurring in R. goughensis are shared with Nesospiza (Neso5, 9, $13-15,17,23)$ and these alleles are found in all three Nesospiza groups in the gene tree (Figures 2 and 3). The estimated minimum number of putatively functional gene copies in Nesospiza (i.e. 4 loci) suggests that the three Nesospiza allele groups are not necessarily locus-specific, despite their divergent clustering. Group 3 may represent a single locus, since only one or two alleles from this cluster occur in each individual. However, this is not the case for $R$. goughensis, where three of these alleles occur in one individual. Two highly supported clusters are seen within Group 2 (Figure 3), which is also the cluster containing the most alleles, suggesting that this cluster is likely to represent more than one gene copy. A likely explanation for the clustering of alleles from different gene loci is the genetic homogenization caused by gene duplication events with subsequent gene conversion.

The highly supported branches of sequences forming Groups 2 and 3 in the gene tree contain only Nesospiza and $R$. goughensis alleles. Although several species were included due to the similarity between their MHCII $\beta$ alleles and those of Nesospiza, the observed divergent clustering of Group 2 sequences could be explained by a lack of closely related species in the analysis. Alternatively, the species-specific clustering of Nesospiza may be attributed to their long divergence time from the other passerines sampled [48]. The deep divergence of Groups 2 and 3, and the clustering of Group 3 close to the distantly related species of common yellowthroat, New Zealand robins, Florida scrub jay, and vegetarian finch, however, provide strong support for preserved ancestral polymorphism. These patterns suggest that extant MHC variation in Nesospiza and $R$. goughensis can be explained by shared ancestral polymorphism during colonisation which has since been maintained. It is possible that the additional variation has been generated by gene conversion events, which is the most likely method of generating variation from the few alleles remaining in a population following a population bottleneck [26].

Amino acid sequences are more similar between Groups 1 and 3 (Figure 4). This could either represent evidence of recombination with the pseudogenes, producing a new group of functional sequences, or perhaps more likely indicate that the pseudogenes resulted from gene duplication events of Group 3 sequences. Copying errors during gene duplication and recombination events may result in non-functional genes (pseudogenes) and the subsequent lack of functional constraint on evolutionary processes (such as mutation) acting on the pseudogenes result in rapid sequence divergence [49]. This is evidently the case for the two presumably nonfunctional alleles, Neso22 and Neso23, which form a well supported group with a red-winged blackbird pseudogene, clustered sister to all the functional passerine sequences. However, some pseudogenes (e.g. red-winged blackbird 


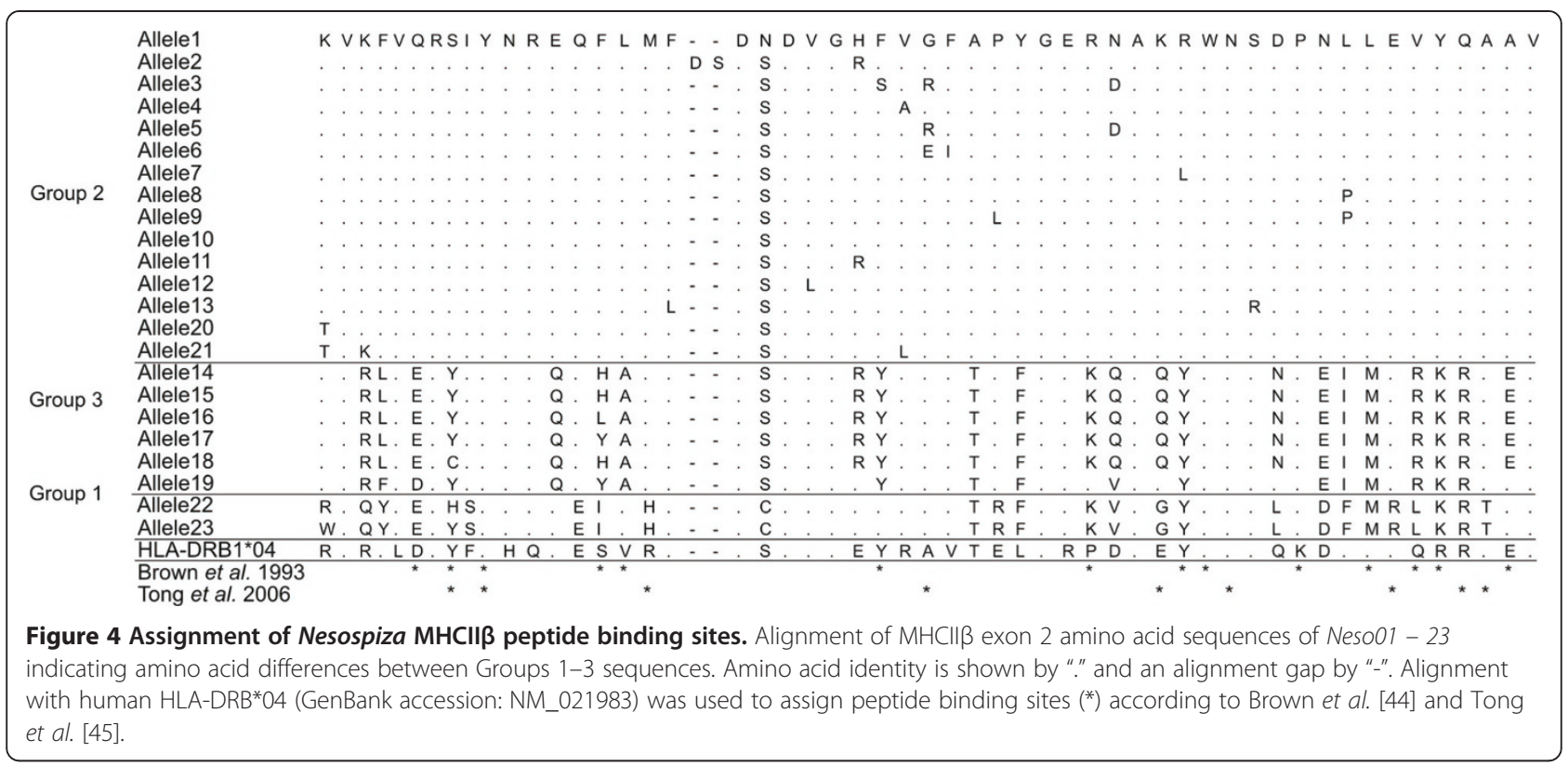

APAF030994 and vegetarian finch PCAY064439) do not show evidence of rapid divergence (Figure 3), perhaps due to ongoing recombination with functional genes that is leading to sequence conservation. Alternatively, there may have been insufficient time for the genes to become highly diverged since they became non-functional.

Selection tests showed no consistent evidences of balancing or positive selection at the PBR or non-PBR regions of MHCII $\beta$ exon 2 in Nesospiza and Rowettia. The short fragment length of our sequences excludes some of the PBR sites, and therefore there is a chance that some sites that may be under selection were excluded from the analyses. However, selection tests were done according to two different PBR characterizations $[44,45]$, and tested on the entire data set as well as all species individually, and the three clusters independently. Ratios of $\mathrm{d}_{\mathrm{N}} / \mathrm{d}_{\mathrm{S}}$ were nonsignificant in all cases (Table 1), and additional selection tests showed weak evidence of selection with only one site likely to be under positive selection (Table 2). New MHC variation can be generated by point mutations or through recombination between alleles, giving rise to a new allele $[26,33]$. The latter process, known as gene conversion, has been documented in some natural avian populations $[22,25,26]$ and has been suggested to be essential in generating genetic variation at MHC after a bottleneck [26]. During gene conversion events, synonymous substitutions may hitchhike with non-synonymous variation [26] and this may be a reason why $d_{N} / d_{S}$ ration tests fail to detect positive selection. We found, however, no evidence of recombination in our data, but recombination can be difficult to verify with short sequences.

Despite the lack of significant evidence for selection, ratios of $d_{N} / d_{S}>1.0$ that we observe in Rowettia and all
Nesospiza populations indicate that the loci are under weak balancing selection, or perhaps more likely, that ancestral balancing selection acted on the loci before colonisation of the islands. Lack of strong positive selection may reflect a decreased pathogen load in both Nesospiza and R. goughensis. Passerines generally are less parasitised by lice and ectoparasites than other avian orders e.g. [50]. This is particularly true of small populations on isolated oceanic islands ( $\mathrm{R}$ Palma pers. comm.). Myrsidea lice occur at extremely low prevalence (6.4\%) across 12 species of Darwin's finches at the Galápagos Islands [50]. On Tristan da Cunha and Gough Island, different louse species (order Phthiraptera) have been found on 20 bird species, including the Tristan thrush (Nesocichla eremita) [51], yet careful inspection of Nesospiza buntings yielded no lice, with hippoboscid flies and feather mites the only ectoparasites (PG Ryan unpubl. data). The absence of parasites could be due to an uninfected founding population ("missing the boat") [52], or subsequent extinction from the host after colonisation. The high level of ancestral polymorphism between $R$. goughensis and Nesospiza suggest that the former is more likely, where a single uninfected founding population colonization both Tristan da Cunha and Gough Island.

Some shortcomings of the cloning and sequencing method employed in the study may result in underestimation of MHC variation. Firstly, the large number of gene copies and the high level of convergence between loci make it difficult to amplify a single MHC locus at a time. Thus, most MHC studies on non-model vertebrates amplify alleles from multiple gene copies simultaneously. This increases the risk of chimera formation during the PCR, which in turn 
leads to overestimation of levels of gene recombination [53]. In addition, PCR products are prone to point mutations e.g. [54], although these are relatively easy to detect since mutation rates are relatively low and are unlikely to occur in more than one sequence $[55,56]$. In this study, we compensate for these problems by only accepting alleles that occur in at least two individuals e.g. $[57,58]$. Secondly, the amplification of a multi-gene family is necessarily problematic since not all loci and not all alleles at a locus will be detected using a single primers set. The primers employed in this study were designed for non locus-specific amplification of exon 2 of MHCII $\beta$ in zebra finch (Taeniopygia guttata) [59] and have been successfully employed in other passerine MHC studies ( $\mathrm{H}$ Westerdahl pers. comm.). A regression analysis of the number of clones sequenced per individual found that more individuals and sequences will be necessary to estimate true $\mathrm{MHC}$ variation per individual. Finally, sequences were obtained for only half of the variable MHCII $\beta$ exon 2 gene. Although not all the variation has been analysed in this study, this is often the case with such complex multi-gene systems [58] and does not preclude our finding of ancestral polymorphism between species and within the Nesospiza species complex. More comprehensive studies of population level variation of $\mathrm{MHC}$ would require that more individuals and sequences were analysed. However, the present study focuses on selection and levels of shared polymorphism, and for such analyses the present data is sufficient.

\section{Conclusions}

The extent of shared alleles and ancestral polymorphism between Nesospiza and $R$. goughensis suggests that both originated from the same colonization wave. We find that similar or the same alleles are maintained between species due to the recent species divergence and low levels of (local) selection acting on PBR. The additional variation found within the Nesospiza species complex may be due to gene conversion, which is likely the most prominent mechanism for generating new variation after a bottleneck event [26]. The extant genetic variation is not likely to change rapidly, unless there is a drastic geographic or environmental change leading to strong selection at the MHC. One such situation would be the introduction of pathogens, since populations with low MHC diversity are often more susceptible to novel pathogens $[35,60]$. In the absence of strong selection, $\mathrm{MHC}$ is expected to diverge over time between islands and populations due to drift, with the generation of new haplotypes through point mutations or gene conversion. Ongoing gene flow between populations and subspecies on Inaccessible Island can maintain genetic variation to some extent. The potential role of MHC dependent sexual selection [22,61] to drive divergence between populations even further remains open to study, and would require wider sampling over the entire geographic range to cover the details of geographicand species-specific variation.

\section{Methods \\ Sampling}

Buntings were mist-netted or caught with hand nets at Inaccessible, Nightingale and Gough Islands during September 1999 - February 2000, with additional samples from Inaccessible Island collected in September November 2004 [37,38]. No extant Nesospiza species occur on Tristan Island. Brachial vein blood samples were collected and stored in EDTA or lysis buffer. Two to three individuals were chosen to represent each population (Figure 1; Inaccessible: $3 N$. a. acunhae, $2 N$. a. fraseri, $2 N$. a. dunnei, $3 N$. a. hybrid; Nightingale: $2 N$. questi, $2 N$. wilkinsi; Gough Island: 2 R. goughensis).

\section{DNA extraction and amplification}

DNA was extracted from whole blood by standard phenol:chloroform methods [Sambrook]. The primers 2zffw1 (5' TGT CAC TTC AYK AAC GGC ACG GAG 3') and 2zfrv1 (5' GTA GTG TGC CGG CAG TAC GTG TC 3’), previously designed for the zebra finch (Taeniopygia guttata) [59], were used to amplify $159 \mathrm{bp}$ of MHCII $\beta$ exon 2. These primers are not locus-specific and amplify exon 2 of multiple copies of the MHCII $\beta$ gene. Amplifications were performed in $10 \mu \mathrm{l}$ volumes, each containing $5 \mu \mathrm{l}$ QIAGEN Multiplex PCR Master Mix, 10 pM of each primer, and 10 ng of template DNA. PCR cycling conditions involved an initial denaturing step of 15 minutes at $95 \mathrm{C}$, followed by 35 cycles of 30 seconds at 94C, 1 minute 30 seconds at $64 \mathrm{C}$ and 1 minute 30 seconds at $72 \mathrm{C}$.

\section{Cloning and sequencing}

PCR products of all individuals were cloned using the TOPO TA Cloning ${ }^{\circledR}$ kit (Invitrogen). Vectors (pCR ${ }^{\circledR}$ 2.1-TOPO ${ }^{\circledR}$ ) with inserted PCR product were used to transform chemically competent Escherichia coli cells (OneShot $\left.{ }^{\circledR}\right)$, according to the manufacturer's instructions. Transformed cells were cultured on S.O.C medium (Invitrogen) for one hour in a shaking incubator at $37 \mathrm{C}$ and then incubated overnight at $37 \mathrm{C}$ on LBmedium supplemented with $50 \mu \mathrm{g} / \mathrm{ml}$ Ampicillin and $50 \mu \mathrm{l}$ of X-gal $(40 \mathrm{mg} / \mathrm{ml})$. For each sample 30 positive colonies were picked with a sterile toothpick, diluted in $100 \mu \mathrm{l}$ Sabax water (Adcock Ingram) and used directly as DNA template for PCR. Amplification reactions contained $2 \mu \mathrm{l}$ QIAGEN Multiplex Master Mix, 10 pM each of M13 forward and M13 reverse primers (included in the kit), and $2 \mu \mathrm{l}$ of the colony diluted in Sabax water. The same PCR cycling conditions were used as before (see above). All clones were sequenced in both directions on an ABI Prism 3100 capillary sequencer (Applied 
Biosystems). A total of $12-29$ clones were successfully sequenced per individual (average $=22.88)$.

\section{Data analysis}

Nucleotide sequences were edited and aligned using CLC Main Workbench 5.0.2 (CLC Bio). To avoid including false haplotypes due to artefacts arising during PCR (e.g. recombinant chimeric sequences), sequences were only accepted if they were present in two or more individuals $[56,62]$ (396 of 508 sequences were accepted and these represented 23 different alleles; Additional file 1 Table S1). Due to the large number of sequences excluded with this stringent method, we followed Anmarkrud et al. [5] suggestion to identify additional true alleles and evaluated whether the excluded sequences were $>1.5 \%(\sim 3 \mathrm{bp})$ different from any of the sequences that were identified as possible alleles. Only two of the excluded sequences differed with $>1.5 \%$ and since so few alleles would not affect the results we decided not to include them in the analyses.

The nucleotide diversity $(\pi)$ was calculated using DnaSP 5.0 [63]. Sequences were verified as MHC alleles using the BLASTN 2.2.24 algorithm [64] available through the National Center for Biotechnology Information (NCBI). Of the 23 alleles identified, 21 (NesoO1 - 21) showed high identity (87-96\%, with coverage of $80-98 \%$ ) to known passerine MHCII $\beta$ coding genes, and two alleles (Neso22 and Neso23) showed high identity (92-93\%, with $98 \%$ coverage) with passerine pseudogenes (Figure 3). This suggests that Neso22 and Neso23 are non-functional, thus they were excluded from the selection tests.

A regression analysis was performed to determine if the number of sequences obtained for each individual effectively sampled the total number of alleles. For each individual, a random subset of the alleles obtained was sampled and the number of alleles in the subset counted. This was repeated 100 times each for a subset of 5, 10, 15, 20 and 25 (restricted by the number of sequences obtained for each individual). As sampling approaches the maximum number of alleles in the population, the number of alleles found in increasing subset sizes will plateau.

Nucleotide positions associated with the PBR were assigned according to the PBR regions determined for the human antigen binding region by two different studies $[44,45]$. Selection was tested using the ratio of nonsynonymous $\left(\mathrm{d}_{\mathrm{N}}\right)$ to synonymous $\left(\mathrm{d}_{\mathrm{S}}\right)$ substitutions $\left(\mathrm{d}_{\mathrm{N}} / \mathrm{d}_{\mathrm{S}}=\omega\right)$. Under strict neutrality $d_{N}=d_{S}$, while regions under balancing selection are expected to undergo more nonsynonymous substitutions and regions under directional selection more synonymous substitutions. The parameter $\omega$ was calculated in MEGA 4 [65] using the method of Nei and Gojobori [66] with Jukes Cantor corrections and 1000 bootstrap replicates. A z-test [66] was used to determine the probability of selection by comparing the selection parameter, $\omega$, against a null hypothesis of strict neutrality
$\left(\mathrm{d}_{\mathrm{N}}=\mathrm{d}_{\mathrm{S}}\right)$. Standard selection tests (Tajima's D, Fu \& Li's F* and Fu \& Li's D*) were calculated in DnaSP 5 [63]. Substitution rates, $\omega$, and the probability of positive selection on $\mathrm{PBR}$ and non-PBR regions, were compared to results from New Zealand and Chatham Island robins (Petroica australis and Petroica traverse) [34,35], Hawaiian honeycreepers (Drepanidinae) [46], common yellowthroat (Geothlypis trichas) [43], and house sparrow (Passer domesticus; values calculated using sequences from GenBank).

In a second test of selection, the maximum likelihood method implemented in CODEML in the Phylogenetic Analysis by Maximum Likelihood package (PAML 3.14) $[67,68]$, was used to identify the sites under selection. Likelihood ratio tests in CODEML were used to test neutral models and models of selection. In a first comparison, a neutral model M1a $\left(\omega_{0}<1, \omega_{1}=1\right)$ was tested against M2a, a model for positive selection $\left(\omega_{2}>1\right)$. Model M1a assumes that sites are either conserved or under purifying selection (i.e. removed from the population) $\left(\omega_{0}<1\right)$, or selectively neutral $\left(\omega_{1}=1\right)$. Model M2a considers a third class of sites where sites may be under positive selection $\left(\omega_{2}>1\right)$. A second comparison tested a neutral model M7 $(0<\omega<1)$ against a model for positive selection, M8 $(0<\omega<1, \omega>1)$. Model M7 is based on a $\beta$ distribution and estimates $\omega$ as a value between 0 and 1. In M8, $\omega$ is estimated directly from the data for one class of sites which allows for $\omega>1$. Both these tests are used routinely to identify sites under selection [69]. The best-fit model was determined using a likelihood ratio test for each model comparison, thus the likelihood of positive selection could be evaluated [70]. The difference in likelihood values of the null model (M1a, M7) and the alternative model (M2a, M8) was compared with the $x^{2}$ distribution. Degrees of freedom were calculated as the difference in the number of parameters for each test. The Bayes Empirical Bayes method, implemented in CODEML, was used to calculate the posterior probability for each site class for the M2a and M8 models. A site is likely to be under positive selection when the posterior mean of $\omega>1$ [68].

To determine the phylogenetic relationship between the 23 Nesospiza alleles a Neighbour-Joining (NJ) tree was constructed in MEGA 4 [65] assuming homogenous substitution patterns among lineages and uniform rates among sites. A consensus tree was computed from 10000 bootstrap replicates in MEGA 4 [65] using a 75\% consensus cut-off value. All subsequent phylogenetic analyses were conducted in MrBayes v 3.1.2 [70]. A concatenated data set comprising MHCII $\beta$ sequences from several passerines obtained from GenBank (Figure 3) was analysed with all Nesospiza alleles (Neso01 - Neso23). The passerine species most closely related to Nesospiza, chosen as the top ten hits for each Nesospiza allele using BLAST, and several other passerine species (chosen to represent passerine diversity), were used 
for the phylogenetic analyses. Sequences were only included if there was sequence alignment of more than $100 \mathrm{bp}$, thus some species (e.g. Poephila acuticauda) identified to be in the top ten closest matches to one of the Nesospiza alleles were not included. This cut-off was made to ensure a robust result from the phylogenetic analysis.

The best model for nucleotide substitution was chosen using the Akaike Information Criterion (AIC) [71] as determined by jModelTest [72,73] for each codon position independently (Position 1: TIM3ef + I + G; Position 2: TVM + G; Position 3: TPM2uf $+\mathrm{G})$. Divergent zebra finch sequences were chosen as a root for passerine MHCII [60]. MrBayes was run for 3 million generations with four incrementally heated chains. Trees were sampled every 3000 generations, with a $10 \%$ burn-in. A consensus tree and posterior probabilities were calculated from the sampled trees. The average standard deviation of split frequencies between two simultaneous runs was monitored to confirm convergence.

The RDP3 Beta 27 [74] package was used to test for signatures of recombination using multiple algorithms simultaneously: RDP [75], GENECONV [76], BootScan [77], MaxChi [78], Chimaera [79], and 3Seq [80]. The default settings were used, and the significance level was set to 0.05 . Bonferroni corrections were applied for multiple comparisons [81].

\section{Additional material}

GenBank accession numbers of non-Nesospiza sequences used in the present study: L42334 - L42335, U23968 U23969, U23967, U23970, U23971, AJ404371 - AJ404 376, U24405, AY437900 - AY437912, AY428561 - AY42 8568, AY258333 - AY248335, AY428569, U23958 - U2 3966, U23972, U23973, U23975, XM_002192161, XM_0 02193356, XM_002196138, XM_002197722, XM_00219 8130, XM_002198161, XM_002199709, XM_ 0022002 57, AF165156 - AF165157, AF165159, Z74424 - Z74428, AY064425, AY064439, AY064451, GQ247601 - GQ2476 06, GQ247608 - GQ247609, GQ247613 - GQ247614, GQ 247616 - GQ247622, GU390288 - GU390291, AY518171 AY518183, AY583092 - AY583094.

\section{Additional file}

Additional file 1: Table S1. List of 23 major histocompatibility complex class II $\beta$ (MHC\| $\beta$ ) exon 2 Nesospiza bunting sequences used in the present study.

\section{Authors' contributions}

AJvR carried out the molecular lab work, statistical analyses, and drafted the manuscript. BH, PB, and PGR conceived of the study and participated in its design. $\mathrm{BH}$ participated in the coordination of the study and helped to draft the manuscript. All authors read and approved the final manuscript.

\section{Acknowledgements}

We thank Coleen Moloney and Cliff Dorse for assistance with collecting samples, Helena Westerdahl for providing primers, and Martin Stervander for alignment and processing of sequence data. Ricardo Palma from the Museum of New Zealand, Te Papa Tongarewa, gave helpful comments based on his work on Phthiraptera in avian systems. This work received financial support from a European Union IRSES grant (PIRSES-GA-2008-230799) and South Africa/ Sweden Bilateral funding (348-2008-6131) to Paulette Bloomer, Bengt Hansson, and Peter Ryan; and research grants from the Crafoord Foundation and the Swedish Research Council to Bengt Hansson.

\section{Author details}

${ }^{1}$ Molecular Ecology and Evolution Program, Department of Genetics, University of Pretoria, Private bag X20, Hatfield 0028, South Africa. ${ }^{2}$ Percy FitzPatrick Institute of African Ornithology, DST/NRF Centre of Excellence, University of Cape Town, Rondebosch 7701, South Africa. ${ }^{3}$ Molecular Ecology and Evolution Lab, Department of Biology, Lund University, SE-22362 Lund, Sweden.

Received: 7 November 2011 Accepted: 13 July 2012 Published: 15 August 2012

\section{References}

1. Dlugosch KM: Parker: Founding events in species invasions: genetic variation, adaptive evolution, and the role of multiple introductions. MolEcol 2008, 17:431-449.

2. Lande $R$, Shannon S: The role of genetic variation in adaptation and population persistence in a changing environment. Evolution 1996, 50:434-437.

3. Robertson A: Selection for heterozygotes in small populations. Genetics 1962, 47:1291-1300.

4. Oliver MK, Piertney SB: Selection maintains MHC diversity through a natural population bottleneck. MolBiolE 2012, in press.

5. Anmarkrud JA, Johnsen A, Bachmann L, Lifjeld T: Ancestral polymorphism in exon 2 of bluethroat (Lusciniasvecica) MHC class II B genes. J EvolBiol 2010, 23:1206-1217.

6. Hess CM, Edwards SV: The evolution of the major histocompatibility complex in birds. Bioscience 2002, 52:423-431.

7. Ekblom R, Sæther SA, Jacobsson PAR, Fiske P, Sahlman T, Grahn M, Kålås JA, Höglund J: Spatial pattern of MHC class II variation in the great snipe (Gallinago media). MolEcol 2007, 16:1439-1451.

8. Klein J: Natural history of the major histocompatibility complex. New York: John Wiley \& Sons; 1986.

9. Parham P, Ohta T: Population biology of antigen presentation by MHC class I molecules. Science 1996, 272:67-74.

10. Gaudieri S, Dawkins RL, Habara K, Kulski JK, Gojobori T: SNP profile within the human major histocompatibility complex reveals an extreme interrupted level of nucleotide diversity. Genome Res 2000, 10:1579-1586.

11. Doherty PC, Zinkernagel RM: Enhanced immunological surveillance in mice heterozygous at the $\mathrm{H}-2$ complex. Nature 1975, 256:50-52.

12. Penn DJ, Potts WK: The evolution of mating preferences and major histocompatibility complex genes. Am Nat 1999, 153:145-164.

13. Spurgin $L G$, Richardson DS: How pathogens drive genetic diversity: MHC, mechanisms and misunderstandings. Proc R Soc Lond B Biol Sci 2010, 277:979-988.

14. Takahata N, Satta Y, Klein J: Polymorphism and balancing selection at major histocompatibility loci. Genetics 1992, 130:925-938.

15. Oliver MK, Telfer S, Piertney SB: Major histocompatibility complex (MHC) heterozygote superiority to natural multi-parasite infections in the water vole (Arvicolaterrestris). Proc R Soc Lond B Biol Sci 2009, 276:1119-1128.

16. Penn DJ: The scent of genetic compatibility: sexual selection and the major histocompatibility complex. Ethology 2002, 108:1-21.

17. Richardson DS, Komdeur J, Burke T, von Schantz T: MHC-based patterns of social and extra-pair mate choice in the Seychelles warbler. Proc $R \operatorname{Soc} B$ 2005, 272:759-767.

18. van Oosterhout $\mathrm{C}$ : A new theory of MHC evolution: beyond selection on the immune genes. Proc R Soc B 2009, 276:657-665.

19. Nei M, Rooney AP: Concerted and birth-and-death evolution of multigene families. Annu Rev Genet 2005, 39:121-152.

20. Edwards SV, Grahn M, Potts WK: Dynamics of $M H C$ evolution in birds and crocodilians: amplification of class II genes with degenerate primers. MolEcol 1995, 4:719-729. 
21. Westerdahl $H$, Wittzell $H$, von Schantz: Mhc diversity in two passerine birds: no evidence for a minimal essential Mhc. Immunogenetics 2000 52:92-100.

22. Promerová M, Albrecht T, Bryja J: Extremely high $M H C$ class I variation in a population of a long-distance migrant, the Scarlet Rosefinch (Carpodacuserythrinus). Immunogenetics 2009, 61:451-461.

23. Nei M, Gu X, Sitnikova T: Evolution by the birth-and-death process in multigene families of the vertebrate immune system. PNAS 1997, 94:7799-7806

24. Gu X, Nei M: Locus specificity of polymorphic alleles and evolution by a birth-and-death process in mammalian MHC genes. MolBiolEvol 1999, 16:147-156.

25. Witzell H, Bernot A, Auffrey C, Zoorob R: Concerted evolution of two MHC class II B loci in pheasants and domestic chickens. MolBiolEvol 1999, 16:479-490.

26. Spurgin LG, van Oosterhout C, Illera JC, Bridgett S, Gharbi K, Emerson BC, Richardson DS: Gene conversion rapidly generates histocompatibility complex diversity in recently founded bird populations. MolEcol 2011, 20:5213-5225

27. Takahata N, Nei M: Allelic genealogy under overdominant and frequencydependent selection and polymorphism of Major Histocompatibility Complex loci. Genetics 1990, 124:967-978

28. Klein J: Origin of Major Histocompatibility Complex polymorphism - the transspecies hypothesis. Hum Immunol 1987, 19:155-162

29. Figueroa F, Gunther E, Klein J: MHC polymorphism in the MHC class II of a non-passerine bird, the great snipe (Gallinago media). Nature 1988, 335:265-267.

30. Lawlor DA, Ward FE, Ennis PD, Jackson AP, Parham P: HLA-A and HLA-B polymorphism predate the divergence of human and chimpanzees. Nature 1988, 335:268-271.

31. Bernatchez $L$, Landry C: $M H C$ studies in nonmodel vertebrates: what have we learned about natural selection in 15 years? J Evolution Biol 2003, 16:363-377.

32. Richardson DS, Westerdahl H: MHC diversity in two Acrocephalus species: the outbred great reed warbler and the inbred Seychelles warbler. MolEcol 2003, 12:3523-3529.

33. Bahr A, Wilson AB: The evolution of MHC diversity: Evidence of intralocus gene conversion and recombination in a single-locus system. Gene 2012, 497:52-57.

34. Miller HC, Lambert DM: Gene duplication and gene conversion in class II MHC genes of New Zealand robins (Petroicidae). Immunogenetics 2004 56:178-191.

35. Miller HC, Lambert DM: Genetic drift outweighs balancing selection in shaping post-bottleneck major histocompatibility complex variation in New Zealand robins (Petroicidae). MolEcol 2004, 13:3709-3721.

36. Rand AL: The origin of landbirds of Tristan da Cunha, Nightingale and Inaccessible Islands. Fieldiana Zoology 1955, 37:139-166.

37. Ryan PG, Bloomer P, Moloney C, Grant TJ, Delport W: Ecological speciation in South Atlantic island finches. Science 2007, 315:1420-1423.

38. Jansen van Rensburg A: Resolving the fine-scale population structure of Nesospizabuntings using a genetic multi-marker system. University of Pretoria: MSc thesis; 2011

39. Ryan PG: Taxonomic and conservation implications of ecological speciation in Nesospizabuntings on Tristan da Cunha. Bird Conservint 2008, 18:20-29.

40. Bollmer JL, Vargas FH, Parker PG: Low MHC variation in the endangered Galápagos penguin (Spheniscusmendiculus). Immunogenetics 2007, 59:593-602.

41. Travis EK, Vargas FH, Merkel J, Gottdenker N, Miller RE, Parker PG: Hematology, serum chemistry, and serology of Galápagos penguins in the Galápagos Islands, Ecuador. J Wildlife Dis 2006, 42:625-632.

42. Klein J, Bontrop RE, Dawkins RL, Erlich HA, Gyllensten UB, Heise ER, Jones PP, Parham P, Wakeland EK, Watkins DI: Nomenclature for the major histocompatibility complexes of different species: a proposal. Immunogenetics 1990, 31:217-219.

43. Bollmer $\mathrm{J}$, Dunn PO, Whittingham LA, Wimpee C: Extensive MHC Class II B gene duplication in a passerine, the common yellowthroat (Geothlypistrichas). J Hered 2010, 101:448-460.

44. Brown JH, Jardetzky TS, Gorga JC, Stern LJ, Urban RG, Storminger JL, Wiley DC: Three-dimensional structure of the human class II histocompatibility antigen HLA-DR1. Nature 1993, 364:33-39.
45. Tong JC, Zhang GL, Tan TW, August JT, Brusic V, Ranganathan S: Prediction of HLA-DQ3.2 $\beta$ Ligands: evidence of multiple registers in class II binding peptides. Bioinformatics 2006, 22:1232-1238.

46. Jarvi SI, Tarr CL, Mclntosh CE, Atkinson CT, Fleischer RC: Natural selection of the major histocompatibility complex $(M H C)$ in Hawaiian honeycreepers (Drepanidinae). Mol Ecol 2004, 13:2157-2168.

47. Bonneaud C, Sorci G, Morin V, Westerdahl H, Zoorob R, Wittzell H: Diversity of MHC class I and II B genes in house sparrows (Passer domesticus). Immunogenetics 2004, 55:855-865.

48. Aguilar A, Edwards SV, Smith TB, Wayne RK: Patterns of variation in MHC class II $\beta$ loci of the little greenbul (Andropadusvirens) with comments on MHC evolution in birds. J Hered 2006, 97:133-142.

49. Swanson WJ, Vacquier VD: The rapid evolution of reproductive proteins. Nat Rev Genet 2002, 3:137-144.

50. Palma RL, Price RD: The species of MyrsideaWaterston (Insecta: Phthiraptera: Menoponidae) from the Galápagos Islands, with descriptions of new taxa. Tuhinga 2010, 21:135-146.

51. Hänel C, Palma RL: The lice of the Tristan da Cunha archipelago (Insecta: Phthiraptera). BeiträgeEntomol 2007, 57:105-133.

52. Paterson AM, Gray RD: From Host-parasite co speciation, host switching and missing the boat. In Host-parasiteevolution: General principles and avian models. Edited by Clayton DH, Moore J. Oxford: Oxford University Press; 1997:236-250.

53. Kanagawa T: Bias and artifacts in multitemplate polymerase chain reactions (PCR). J Biosci Bioeng 2003, 96:317-323.

54. Cline J, Braman JC, Hogrefe HH: PCR fidelity of Pfu DNA polymerase and other thermostable DNA polymerases. Nucleic Acid Research 1996, 24:3546-3551

55. Galan M, Guivier E, Caraux G, Charbonnel N, Cosson JF: A 454 multiplex sequencing method for rapid and reliable genotyping of highly polymorphic genes in large-scale studies. BMC Genomics 2010, 11:296

56. Nadachowska-Brzyska K, Zielinski P, Radwan J, Babik W: Interspecific hybridization increases MHC class II diversity in two sister species of newts. Mol Ecol 2012, 21:887-906.

57. Babik W, Durka W, Radwan J: Sequence diversity of the MHC DRB gene in the Eurasian beaver (Castor fiber). Mol Ecol 2005, 14:4249-4257.

58. Babik W: Methods for MHC genotyping in non-model vertebrates. Mol. Ecol. Res. 2010, 10:237-251.

59. Balakrishnan CN, Ekblom R, Völker M, Westerdahl H, Godinez R, Kotkiewicz H, Burt DW, Graves T, Griffin DK, Warren WC, Edwards SV: Gene duplication and fragmentation in the zebra finch major histocompatibility complex. BMC Biol 2010, 8:29.

60. Radwan J, Biedrzycka A, Babik W: Does reduced $M H C$ diversity decrease viability of vertebrate populations? Bio/Conserv 2010, 143:537-544.

61. Agudo R, Alcaide M, Rico C, Lemus JA, Blanco G, Hiraldo F, Donázar JA: Major histocompatibility complex variation in insular populations of the Egyptian vulture: inferences about the roles of genetic drift and selection. Mol Ecol 2011, 20:2329-2340.

62. Lenz TB, Becker S: Simple approach to reduce PCR artefact formation leads to reliable genotyping of MHC and other highly polymorphic loci - implications for evolutionary analysis. Gene 2008, 427:117-123.

63. Librado P, Rozas J: DnaSP v5: a software for comprehensive analysis of DNA polymorphism data. Bioinformatics 2009, 25:1451-1452.

64. Zhang Z, Schwartz S, Wagner L, Miller W: A greedy algorithm for aligning DNA sequences. Journal Comput Biol 2000, 7:203-214.

65. Tamura K, Dudley J, Nei M, Kumar S: MEGA4: Molecular Evolutionary Genetics Analysis (MEGA) software version 4.0. Mol Biol Evol 2007, 24:1596-1599.

66. Nei M, Gojobori T: Simple methods for estimating the numbers of synonymous and nonsynonymous nucleotide substitutions. Mol Biol Evol 1986, 3:418-426.

67. Yang ZH: PAML 4: Phylogenetic analysis by maximum likelihood. Mol Biol Evol 2007, 24:1586-1591.

68. Yang ZH, Wong WSW, Nielsen R: Bayes empirical Bayes inference of amino acid sites under positive selection. Mol Biol Evol 2005, 22:1107-1118.

69. Yang Z, Nielsen R, Goldman N, Pedersen A-MK: Codon-substitution models for heterogeneous selection pressure at amino acid sites. Genetics 2000, 155:431-449.

70. Ronquist F, Huelsenbeck JP: MrBayes 3: Bayesian phylogenetic inference under mixed models. Bioinformatics 2003, 19:1572-1574. 
71. Akaike $\mathrm{H}$ : A new look at the statistical model identification. IEEE T Automat Contr 1974, 19:716-723.

72. Posada D: jModelTest: Phylogenetic model averaging. MolBiolEvol 2008, 25:1253-1256

73. Posada D: Selection of models of DNA evolution with jModelTest. Meth Mol Biol 2009, 537:93-112.

74. Martin DP, Williamson C, Posada D: RDP2: recombination detection and analysis from sequence alignments. Bioinformatics 2005, 21:260-262.

75. Martin D, Rybicki E: Detection of recombination amongst aligned sequences. Bioinformatics 2000, 16:562-563.

76. Padidam M, Sawyer S, Fauquet CM: Possible emergence of new geminiviruses by frequent recombination. Virology 1999, 265:218-225.

77. Martin DP, Posada D, Crandall KA, Williamson C: A modified bootscan algorithm for automated identification of recombinant sequences and recombination breakpoints. AIDS ResHumRetrov 2005, 21:98-102.

78. Maynard Smith J: Analysing the mosaic structure of genes. J Mol Evol 1992, 34:126-129.

79. Posada D, Crandall KA: Evaluation of methods for detecting recombination from DNA sequences: Computer simulations. PNAS 2001, 98:13757-13762.

80. Boni MF, Posada D, Feldman MW: An exact nonparametric method for inferring mosaic structure in sequence triplets. Genetics 2007, 176:1035-1047.

81. Rice WR: Analyzing tables of statistical tests. Evolution 1989, 43:223-225.

\section{doi:10.1186/1471-2148-12-143}

Cite this article as: Jansen van Rensburg et al: Ancestral polymorphism at the major histocompatibility complex $(\mathrm{MHC} \| \beta)$ in the Nesospiza bunting species complex and its sister species (Rowettia goughensis). BMC Evolutionary Biology 2012 12:143.

\section{Submit your next manuscript to BioMed Central and take full advantage of:}

- Convenient online submission

- Thorough peer review

- No space constraints or color figure charges

- Immediate publication on acceptance

- Inclusion in PubMed, CAS, Scopus and Google Scholar

- Research which is freely available for redistribution 